\title{
COMMENT
}

\section{Chemistry and materials science for a sustainable circular polymeric economy}

Vânia G. Zuin $\circledast^{1,2,3 凶}$ and Klaus Kümmerer ${ }^{1,4 凶}$

Chemistry plays a determining role in every stage of the plastic life cycle. We reflect on the challenges and limitations of plastics - their sheer abundance, chemodiversity and imperfect recoverability leading to loss of material - and on the need for chemical and non-chemical approaches to overcome them.

Plastics mold our contemporary way of life - from face masks, gloves and medical testing kits needed to fight COVID-19 (REF. ${ }^{1}$ ), to innumerable other applications. More than 12 billion tons of plastic waste will be produced by $2050\left(\right.$ REF. $\left.^{2}\right)$. To chemically design a truly circular polymeric economy in which this waste is eliminated or recycled, we need new approaches, methods and resources. Various studies have underlined the importance of closing the plastic loop across all sectors of society (which includes industry, governmental and non-governmental organizations, and services) by focusing on more intelligent designs for polymers and the sustainable use of plastics ${ }^{2}$; this typically refers to re-using and recycling the materials or finding longerlasting and environmentally friendly uses for plastics. But what does closing the loop mean when we consider the huge diversity of plastics on the one hand, and the basic thermodynamic laws on the other?

A circular plastics economy alone will not resolve all problems: total material flows have to be taken into account, as well as their complexity and diversity. Truly becoming more sustainable involves less use, thinking about where plastics offer advantages to all stakeholders and where not, and, importantly, not postponing problems to the future by increasing the complexity of materials or downcycling further. Examples of deferring the problem include converting polyethylene terephthalate (PET) from plastic bottles into textile fibres, which only adds an additional loop instead of enabling more cycles; using wood in place of plastics as a construction material in buildings; or remolding mixed plastics into paving blocks that can only be incinerated at the end of their life and will be a source of microplastic in the environment by abrasion.

\section{The diversity and complexity of plastic}

What we simply call plastic is often already a highly diverse mixture of additives and polymers. There are many individual polymers - PET, polystyrene (PS), polyvinyl chloride (PVC) and polyamides, to name just a few - and co-polymers of varied combinations. Many contain not only carbon and hydrogen but also oxygen, nitrogen, chlorine, fluorine or sulfur. Polymers can have mixed building blocks, different stereochemical arrangements of functional groups, or branched or interlinked segments. Most often, they include chemically bound or physically mixed additives to modify basic properties for a given application. To date, more than 10,500 plastic-related additives are in use, such as plasticizers, flame retardants, colouring agents, UV stabilizers, antioxidants, dyes and glitter. Many have gaps in understanding their toxicity to humans and the environment ${ }^{3}$. To complicate matters further, different plastic materials are often used within one product. Even plastics used for simple applications such as food packaging can contain several layers of different polymers as well as additives. Yet in other cases, one single plastic material or specific additive is used in diverse products. During use and at the end of their lives, all of these products and plastics - usually containing additional chemicals such as dyes, metals and degradation products of polymers and additives - are mixed in waste, before recycling, and in the environment. In other words, at all stages of plastics' lifetime and length scales, from atoms to molecules to materials to applications, there is enormous chemodiversity. What are the costs of this highly complicated diversity, and how do we deal with it?

\section{To circulate or not to circulate?}

All mixing is favoured by an increase in entropy. However, that means that separating the components of plastic requires energy and generates unwanted side products: that is, waste may be deferred to the future or another place. Every step of circulating and recycling leads to downgrading and loss of material. Fresh material has to be added to achieve certain grades of recycled plastics or polymers. For example, if there are 
four recycling steps and each one can recover $95 \%$ of material - which is far from reality for most steps only $81 \%$ of a constituent will be regained. Even in the case of pure waste materials, such as PET from water bottles, at least $5 \%$ or more is lost during recycling processes, let alone losses related to collection. Even remolding a native polymer granule induces chemical reactions, lowering the quality of the polymer. Depolymerization requires even more resources (auxiliary chemicals and energy) and generates more waste than remolding.

The steep increase in plastic usage and the everchanging chemodiversity of plastics often does not allow enough time to develop effective and efficient recycling processes and systems. According to thermodynamics, we cannot completely avoid these losses; we can only try to minimize them. The higher the diversity and volume of plastics, and the more the associated energy, substances, material and product flows, and related waste streams, the more we will lose. In contrast to the high chemodiversity of plastics, nature uses only a few structurally closely related basic building blocks, such as glucose or amino acids. Although these building blocks are used everywhere, the associated material flows are local ones. There are no global natural flows of such materials. Nature mostly has geochemical cycles of elements such as carbon, nitrogen, phosphorus and sulfur, based on simple (in)organic compounds only. We need to think about the global circulation of plastics, reducing chemodiversity and downsizing material flows in both space and time, keeping the different kinds of plastics separate.

\section{Box $1 \mid$ Rs for plastic circularity}

\section{Responsibility}

- Take ownership of your own product throughout the whole life cycle beyond the value chain and required function

\section{Reduce}

- Avoid consumption and thereby the amount of waste generated: the first and most effective step towards producing less waste and needing less energy and resources; relates to flows of substances, building blocks, materials and products at all levels, including the following

- Size (tonnage)

- Chemodiversity (complexity of composition, mixing)

- Dynamics (size, composition)

- Space (local, regional, global)

- Time (for example, speed of innovation at the design side versus recycling side and life time)

\section{Reuse (and extend use)}

- Use an item again and again in its original form: this eliminates the need to reprocess materials (whether it is for disposal or for recycling) and reduces dissipative losses

\section{Recycle}

- First, keep form and size (for example, do not cut truck tarpaulin into pieces for bags)

- Second, keep material (remold thermoplastics, avoid thermosets)

- Third, keep the composition (do not add or remove constituents when re-melting)

- Fourth, keep molecules (polymers, additives)

- Fifth, keep building blocks (monomers)

- Sixth, keep atoms (pyrolysis)

- Seventh, keep energy ('thermal recycling', which is in fact combustion)

\section{There are more than $\mathbf{3 R s}$}

Important principles for a circular plastic economy are the 3Rs (reduce, reuse, recycle). However, much more needs to be considered in addition to the 3 Rs (BOX 1). The origin and composition of feedstocks and products; ways to collect, extract and transform them; and the associated risks and benefits, costs and gains, drivers, potentialities, functions, and services must all be regarded as a system ${ }^{4,5}$. These are key criteria for more sustainable plastics that all sectors must assess, communicate and learn about ${ }^{6}$. Therefore, an additional, more overarching $\mathrm{R}$ is needed: responsibility - that is, ownership of products beyond just their intended application, throughout their entire life cycles. To ensure responsibility, dialogues leading to trustful relationships should be established between all stakeholders involved in the plastic life cycle.

New criteria based on chemistry keystones could be considered to improve the circularity of plastics?: simplicity and sufficiency to provide a specific chemical function; and adequacy in time, space, quantity, quality and scale. Developing new and better plastics alone will not be a sufficient contribution of chemistry if we do not simultaneously manage to reduce volume, size, dynamics, complexity and diversity at all levels of the composition of a plastic and at all stages of its life cycle. One approach to support this goal is to not just maximize each functional feature but to intentionally optimize it in the broader context of the entire chemical loop. In other words, materials science and chemistry have to be put into the broader context of sustainability beyond greenness.

\section{The broader perspective}

We must question not only the materials themselves (their origin, characteristics, properties and fates) ${ }^{8}$ but also the processes to obtain or transform them and the services they provide. We must also question where and for whom the high diversity of plastics is beneficial or disadvantageous. Incremental chemical or technical innovations that only postpone challenges to the future are dystopic solutions. Here comes a Fellini moment ${ }^{9}$ : in the closing scene of his film $E$ la nave $v a$, a rhinoceros munches on hay as it rides a lifeboat on a vast ocean. Like the rhinoceros, we are navigating an 'ocean' of plastics, equally immense. Like the rhinoceros, we should long for the sea to dry up to survive. Should we just sit back and observe what happens to us, 'munching' happily? We need to take more action across the entire circular plastic economy! Responsible systems thinking and action are necessary to achieve a happy end to this voyage. This is an invitation to make chemistry and materials science a protagonist in this challenging moment: all members of the crew are responsible for designing much more than new plastics, but also more sustainable systems ${ }^{10}$. And then the ship can sail on ... into a cleaner and calmer future.

1. Yuan, X., Wang, X., Sarkar, B. \& Ok, Y. S. The COVID-19 pandemic necessitates a shift to a plastic circular economy. Nat. Rev. Earth Environ. 2, 659-660 (2021)

2. Chemistry can help make plastics sustainable - but it isn't the whole solution. Nature 590, 363-364 (2021).

3. Wiesinger, H., Wang, Z. \& Hellweg, S. Deep dive into plastic monomers, additives, and processing aids. Environ. Sci. Technol. 55 9339-9351 (2021). 
4. Zuin, V. G. Circularity in green chemical products, processes and services: innovative routes based on integrated eco-design and solution systems. Curr. Opin. Green Sustain. Chem. 2, 40-44 (2016).

5. Kümmerer, K., Dionysiou, D. D., Olsson, O. \& Fatta-Kassinos, D. A path to clean water. Science 361, 222-224 (2018).

6. Zuin, V. G. \& Kümmerer, K. Towards more sustainable curricula. Nat. Rev. Chem. 5, 76-77 (2021).

7. Kümmerer, K., Clark, J. H. \& Zuin, V. G. Rethinking chemistry for a circular economy. Science 367, 369-370 (2020).
8. Williams, C. K. \& Gregory, G. L. High-performance plastic made from renewable oils is chemically recyclable by design. Nature 590 , 391-392 (2021).

9. Fellini, F. E la nave va http://www.britannica.com/biography/ Federico-Fellini (1983).

10. Kümmerer, K. Sustainable chemistry: a future guiding principle. Angew. Chem. Int. Ed. 56, 16420-16421 (2017).

\section{Competing interests}

The authors declare no competing interests. 